Journal of Advances in Sports and Physical Education

Abbreviated Key Title: J Adv Sport Phys Edu ISSN 2616-8642 (Print) |ISSN 2617-3905 (Online) Scholars Middle East Publishers, Dubai, United Arab Emirates Journal homepage: https://saudijournals.com

Original Research Article

\title{
Athletic Pubalgia: A Clinical Reform
}

\author{
Jibran Ahmed Khan ${ }^{1 *}$, Saras Gupta ${ }^{2}$
}

${ }^{1}$ MPT (Sports), Senior Sports Physiotherapist, Lakshya Institute of Shooting \& Sports, Aligarh, U.P, India

${ }^{2}$ BPT, Physiotherapist, Instant relief pain management clinic, New Delhi, India

DOI: $10.36348 /$ jaspe.2022.v05i01.001 | Received: 06.12.2021 | Accepted: 10.01.2022 | Published: 15.01.2022

*Corresponding author: Jibran Ahmed khan

MPT (Sports), Senior Sports Physiotherapist, Lakshya Institute of Shooting \& Sports, Aligarh, U.P, India

\section{Abstract}

This review sums up the current information in regards to anatomy, biomechanics, clinical presentation, differential findings, Imaging, physiotherapy treatment, pre-operative and post-operative recovery of athletic pubalgia. The athletic pubalgia is more common in male athletes as compare with female athletes. Most usually, men experience the ill effects of athletic pubalgia that expects athletes to perform sharps turns, cutting, turning and tedious kicking. The debilitating of posterior inguinal wall is the offender behind athletic pubalgia. It is caused by immoderate and disproportionate application of shear forces through the normal attachments of the abdominal muscles and hip adductors. At present there is no unanimity of assessment on the constituents of this analysis. Since, it is not difficult to characterize a reasonable finding that depends on actual assessment, the techniques, for example, symptomatic ultrasonography and MRI are regularly utilized essentially to offset the presence of different conditions. This is high an ideal opportunity for committed research work on outcome estimation of chronic athletes and furthermore putting together its prosperity totally with respect to reasonable for getting back to sports action. Furthermore, it is likewise needed to have point by point depictions of the techniques, surgical anatomy, conventional treatment and recovery. The data acquired from such an research work will help in understanding pathogenesis of athletic pubalgia in a superior manner, develop proof arranged screening and check for better surgical techniques and successfully oversee both post-surgical and conservative recovery.

Keywords: Sports Hernia, Abdominal Muscles, Hip Adductors, Athletic Pubalgia, Gilmore's groin.

Copyright (C) 2022 The Author(s): This is an open-access article distributed under the terms of the Creative Commons Attribution 4.0 International License (CC BY-NC 4.0) which permits unrestricted use, distribution, and reproduction in any medium for non-commercial use provided the original author and source are credited.

\section{INTRODUCTION}

Athletic pubalgia, otherwise called sports hernia, Gilmore's groin, it is normal in sports that expect athletes to perform tedious kicking, turning or turning at high rates, like cricket, base-ball, tennis, and most generally soccer and hockey [1-5]. The term athletic pubalgia alludes to chronic inguinal or pubicregion torment in athletes that is available with effort just and not logical preoperatively by a tangible hernia or other clinical analysis [6]. In these athletes the inguinal aggravation can be one-sided or respective, its area can shift and it tends to be related with average thigh manifestations, most regularly identified with the hip adductors [3]. Once in a while it is the after effect of an acute injury, because of tedious eccentric overburden to the abdominal wall pelvis stabilisers[9]. It happens in $5 \%$ to $28 \%$ of athletes [8] most normally found in male athletes[9] albeit 2\% of female athletes who have been diagnosed with a hernia have additionally been found to have athletic pubalgia [9].
Inconsistencies among male and female athletes determined to have athletic pubalgia identifies with physical contrasts instead of interest [10]. Females have a more extensive pelvis, making it more steady, which brings about more effective conveyance of powers to the lower body [11] and strength contrasts between the sexual orientations additionally add to the expanded rate in the male population. Literature is loaded up with confusing and regularly clashing data in regards to its motivation, clinical show, determination, and treatment of groin torment in athletes $[1,4,6]$. The confounding show makes it every one of the more significant that clinicians ought to have a sharp information on the condition, as sports hernia could without much of a stretch be unseen in the differential of groin pain.

\section{Anatomy and Biomechanics}

The main muscles embedding at or close to the pubis are the rectus abdominal which joins with the transverse abdominis to frame the conjoined tendon. 
Opposite these two muscles and straightforwardly contradicting their powers, is that the is that the adductor longus. Every rectus abdominis emerges from the unrivaled part of the pubic symphysis, with unique excellence frequently ready to be made between lateral and a medial head on the two sides. Inferiorly, the medial head mixes with its contralateral individual; nonetheless, superiorly the medial heads wander and are isolated by the linea alba. The rectus abdominis has a sheet like setup [12], with at least three intramuscular tendinous convergences, one at the level of the umbilicus, other at the xiphoid tip, and other between these two convergences. Sometimes, a fourth crossing point might be available mediocre compared to the umbilicus. The convergences stretch out through just to the anterior surface of the muscle, where they mix with the foremost rectus sheath. This last structure covers the total front part of every rectus abdominis and appends onto the periosteum of the pubic bone foremost and adjoining the rectus abdominis beginning. The adductor muscle longus and adductor muscle brevis have a broad insertion onto the femur. Joined with the inclusion of the gracilis onto the tibia, these three ligaments meet superiorly and get a beginning near one another on a restricted piece of the pubic body only horizontal to the symphysis. The adductor muscle tendon has its starting point straightforwardly in accordance with the beginning of the more superiorly positioned tendon of the rectus abdominis, with the shallow strands of these two ligaments in direct congruity, flowing over the pubic crest. Additionally, posteriorly and somewhat along the side lies the beginning of the adductor muscle brevis. A portion of the medial strands of the adductor muscle longus and adductor muscle brevis tendon append straightforwardly onto the symphyseal capsular tissues and intraarticular plate[13]. At the point when a athletes experiences a athletic pubalgia, these contradicting powers (up pull from conjoined tendon and descending draw from adductor longus) cause interruption of the muscles at their inclusion site (pubis), prompting groin torment. Essentially what happens is that in dreary kicking, turning or turning at high paces the conjoined tendon apply up power and pivots the trunk, and the adductor muscle longus apply descending power and pivots the upper leg. The utilization of this exorbitant measure of power in an imbalanced way causes athletic pubalgia. Regularly, the core muscles are less evolved than the lower limb muscles and disturbance excessively includes the conjoined tendon prompting athletic pubalgia.

\section{Signs and symptoms}

The beginning is generally guileful yet sometimes an underlying abrupt 'tearing' sensation is available. Torment is work out related that is generally arranged over the lower sidelong edge of the rectus abdominis and may transmit toward the testis, suprapubic locale or adductor muscle longus beginning which get disturbed by abrupt speed increase, cutting and kicking, turning, sit-ups, hacking or wheezing [14,
$15]$. Torment normally endures for a little while after a game and there might be firmness and trouble in getting up the next day. Target actual assessment discoveries are typically inadequate[16]. Confined delicacy is there at or simply over the pubic crest on the influenced side and torment at this area is normally reported about opposed sit-ups. Palpation for a positive inguinal hack impulse is normally either negative or dubious and an unpretentious lump in the skin surface shape over the influenced inguinal area can every so often be seen when the patient is seen from a higher place while standing. Opposed hip adduction development is agonizing and the adductor 'squeeze test' is positive in supine as well as 90 degree hip flexed positions. Obturator nerve pressure is analyzed by obtuseness to stick prick sensation in a trademark dissemination along the medial part of the thigh.

\section{Findings}

The base rules needed for a conclusion of sports hernia include: a clinical setting of constant groin torment which creates during exercise, is situated over the lower parallel edge of the rectus abdominis with or without radiation to the testis or adductor longus beginning, and is regularly exasperated by abrupt speed increase, cutting and kicking, turning, sit-ups and hacking or sniffling; Unpretentious yet steady actual assessment discoveries (Actual assessment won't track down a real hernia, despite the fact that the vast majority of these signs and symptoms lead medical services experts to accept that one is available) $[10,17$, 21]. The Valsalva move, alongside muscle testing of the hip adductors, rectus abdominis, rectus femoris, and iliopsoas, will deliver some inconvenience [18], careful differential finding, to manage different reasons for groin torment and a suitable imaging highlights. These measures should be all the while present, on the grounds that: (A) there are various other possible reasons for groin torment in athletes; and (B) asymptomatic direct inguinal hernias are normal in everybody[22].

\section{Differential Findings}

\section{- $\quad$ Adductor Longus Tendinosis}

It regularly happens with constant redundant abuse of the adductor longus [23]. Adductor tendinopathy is for the most part identified with a rectus abdominis-adductor longus aponeurotic injury. In any case, essential adductor muscle tendon wounds additionally happen, which can be turned around with moderate treatment. The proximal myotendinous intersection is that the most continuous area of wounds. Torment is in the upper average thigh that is more regrettable with opposed adduction of the hip. Actual assessment may uncover delicacy on the proximal ligament. MRI discoveries incorporate thickening of the adductor muscle longus tendon on the suggestive side, intratendinous tear, calcification, distal adductor strain and ligament separation. 


\section{- Osteitis Pubis}

It is accepted to result from insecurity of the pubic symphysis in light of ongoing tedious shear and interruption wounds and lopsided pliable pressure from the muscle connections of the pubic symphysis. The resultant change in biomechanics may deliver a fiery reaction, with osteitis and periostitis. Beginning of agony over the pubis that might cause alluded torment in the suprapubic area, adductor beginning, and crotch. Radiographs of the pubic symphysis might show subchondral sclerosis, symphyseal abnormality, and bone resorption [24]. MRI show diffuse marrow edema stretching out from the subchondral plate and regularly including both pubic bodies [25, 26]. Furthermore, periostitis, disintegrations, abnormality in articular surface, foremost and back osteophytes, and subchondral sores may likewise be seen on MRI [27]. The myotendinous connections are protected except if there is concurrent tendinopathy [28]. The marrow edema in osteitis pubis stretches out across the whole front-back measurement of the pubic body.

\section{- Hockey Goalie-Baseball Pitcher Syndrome}

Groin torment in this surprising disorder results from an epimysial or myofascial herniation of the adductor longus muscle belly a few centimeters from the site of its pubic attattachment [29]. Several researchers have recommended a connection with constant dreary pressure at locales of neurovascular penetration [30]. Intense beginning of torment is typically capable by patients in this condition, which might be steady or may discontinuously increment in the wake of extending. Actual assessment regularly prompts the distinguishing proof of a site of agony distal to the symphysis pubis, over the herniated muscle. Imaging discoveries shows edema of the adductor longus muscle gut, which might conceivably possess a focal situation in the herniated region, and a central muscle swell characteristic of herniation[31].

\section{- Pubic Stress Fracture}

Weariness breaks are generally found in athletes, particularly in marathon runners who should reflect strange ongoing dreary pressure that outperform the bone's capacity to rebuild itself. Inferior pubic ramus is the most well-known site of pelvic pressure cracks; notwithstanding, stress cracks of the femoral neck are more predominant and furthermore may cause crotch torment [32]. Risk factors for pressure crack incorporate female sex, lack of healthy sustenance, and changes in preparing program that place more noteworthy measure of weight on the bone. Patients generally present with a steady beginning of agony, which, at an underlying period of injury, is irritated by practice and mitigated by rest. On the off chance that agony endures in any event during rest, it demonstrates a further developed period of injury. On palpation delicacy is normally present over pubic ramus [33]. MRI discovering shows spaces of marrow edema got with liquid delicate successions. There is likewise a likelihood that periosteal edema or calluses and edema in the neighboring muscles and delicate tissues are available sometimes.

\section{- Septic Arthritis-Osteomyelitis}

Infection of the pubic symphysis is normally seen in post pregnancy female patients, patients with pelvic malignancies and patients exposed to coordinate instrumentation of the pubic symphysis yet it is additionally found in competitors without such danger factors[34]. The contamination is regularly brought about by Staphylococcus aureus and may result from hematogenous spread as opposed to coordinate immunization. Patients typically present with fever and an intense beginning of pubic agony that can transmit upto the crotch. The radiographic and MRI provisions of pubic septic osteomyelitis and osteitis pubis are indistinguishable, and clinicians might rely upon the span of indications and the presence of hazard components to assist them with recognizing these conceivable outcomes. In the event that the determination isn't clear, a biopsy test is wanted.

\section{- Acetabular Labral Tear}

There is finished cross-over between the clinical signs of athletic pubalgia and the pathologic conditions related with the hip. A portion of the hip issues which might bring about groin torment are osteoarthritis, synovitis, intra-articular bodies, and acetabular labral tears. The labrum's foremost better part is very helpless than wounds, especially during outer revolution and hyperextension [35]. The labrum is nearly ineffectively vascularized and its foremost better angle is really considered than be especially powerless. Labral wounds incline the hip to paced articular tendon misfortune, particularly close to the tear, a condition that is viewed as the reason for modifications and unsteadiness in contact powers of the articular cartilages [34]. Strange morphology and sign power of the labrum at routine MRI of the hip are characteristic of the finding. Paralabral sores connected with acetabular labral tears can be plainly seen on the pictures acquired with liquid touchy arrangements.

\section{Imaging}

Many investigations recommend that X-ray has less capacity to recognize a athletic pubalgia and is simply beneficial to dispose of the elective determinations; anyway others have proposed that clinical athletic pubalgia quite often show anomalies on MRI [36-40]. The two plainly seen examples of injury include horizontal rectus abdominis/adductor aponeurtoic injury simply close to the midline rectus abdominis/adductor aponeurotic plate injury ${ }^{7}$ and outer inguinal ring. Albeit some level of pubic marrow upgrade is by and large detailed, these discoveries don't predict which patients will acquire from the surgery. 


\section{Physiotherapy Management}

The fundamental point of any treatment in the first class athletes is really to lessen the all-out time the competitor is away from their sporting activity and to upgrade the player's accessibility for the group. Screening athletes is of prime significance particularly searching for changes in stance, stride and adaptability. The pertinence of any screening program is that it is useful, repeatable and forestalls the repeat or beginning of injury. For the overall specialist, in the outpatient setting, pertinent parts of evaluation are figured to analyze the versatility around the hip joints, posterior pelvic tilt appraisal, adductor muscle 'squeeze' test, proof of lumbar lordosis and gluteal control. When the screening evaluation is finished, athletes should go through a thorough preparing planned explicitly for the person. This early piece of a 'useful restoration program' should be instructed before any medical procedure so the athletes has total comprehension of what is generally anticipated from them soon after any surgical procedure.

Accentuation should be put on the balancing out job of the typically disregarded transverse abdominis, internal obliques, gluteals and adductors play as far as stance and practical development. In the late preoperative/early postoperative stage, stress should be laid on keeping up with the security in the neutral spine position. This requires time and a definite clarification of what is required during what seem, by all accounts, to be very straightforward activities for the 'poorly educated' patient. All through the entire recovery program it is applicable to make any restoration function as much sports explicit as could be expected. It should be focused on that the clinical group has brilliant comprehension of the requests of the sports $^{41}$. Proper preoperative and postoperative consideration should permit a full re-visitation of movement inside only 4 to about two months from the date of a surgery. In reality the accentuation should be on the requirement for a nearby contact between the treating clinicians and the physiotherapy group for the preoperative and postoperative recovery.

\section{Surgery}

Before athletic pubalgia surgery is suggested to a patient, the athlete is assured of the proper diagnosis - athletic pubalgia - and that non-surgical management has been offered and ineffective. Once these criteria have been satisfactorily met, then athletic pubalgia surgery is a reasonable option. First, athletes are given a option of anesthesia. Local anesthesia, general or regional anesthesia are the generally available options. Athletic pubalgia surgery can be done with all 3 types so the method of anesthesia is an individual's choice. Athletic pubalgia surgery centers on stabilizing the insertion of the conjoined tendon at the pubic tubercle. Additionally, a superficial nerve is taken away because this nerve can get caught up in the post-surgery scar tissue and mimics the symptoms of a athletic pubalgia. Also, a thin and pliable mesh is placed by the surgeon over the repair and extends along the inguinal canal as this further strengthens the inguinal floor and supports the athletic pubalgia repair. The surgeon makes an incision $4-5 \mathrm{~cm}$ in length. Local anesthetics are generously given before the closing of the incision to cut down on post-surgical discomfort. The initial athletic pubalgia surgery does not take care of the discomfort at the adductor insertion site. If the conjoined tendon is sufficiently supported by the athletic pubalgia surgery, adductor discomfort is almost uniformly treated with postop rehabilitation. Rarely does the adductor needs operative release. Other surgical technique involve a altered Bassini repair, in this technique the transversal fascia from the deep to superficial ring was released. Intraoperative, all athletes were diagnosed for thinning and a variable degree of bulging of the posterior inguinal wall. The conjoined tendon used to be attached to the inguinal ligament in a double-layer continuous suture, as against the interrupted original Bassini repair, with a running 2/0 prolene suture [42]. A concurrent percutaneous adductor release was done by an orthopedic surgeon (G.D.) in all the cases. A bilateral tenotomy was done with complete transection of the epimysial fibres of the adductor longus tendon which is $2 \mathrm{~cm}$ from the pubic insertion. For athletes who have persistent adductor symptoms despite post-operative rehabilitation or for those whose main complaint focused on the adductor non-operative options do exist. Adductor issues that are not responsive to rehabilitation are generally treated with Platelet-Rich Plasma (PRP) injection. PRP is an office based procedure that includes drawing a tube of blood from the athlete's body. The blood is then spundown and the platelet rich plasma is drawn out of the vial. Under aseptic conditions and the use of local anesthesia, the adductor muscle insertion is directly injected with the platelets rich plasma. Post-procedure rehabilitation is continued and it is rare that a second injection is required. This technique has replaced operative release of the adductor insertion.

\section{Pre-Operative Recovery}

Pre-operative recovery includes a) Individual postural evaluation for the premise of restoration program that includes specific tests for ROM of Hip and Spine, myofascial pathways, muscle length, strength tests for Gluteus medius, Transversus abdominis and so forth, separated muscle strength and isokinetic testing of pelvic control b) Training of postoperative exercise program as itemized in first seven day stretch of recovery program. c) Pre-restoration fortifying system for gluteus medius, cross over abdominis, gluteus maximus, erector spinae, hip flexors, horizontal abdominis and hamstrings. d) Powerful steadiness of stance in practical movement and standing/sitting. This will incorporate re-training of hypotonic and hypertonic muscle tissue around the pelvis. e) Increment portability/protracting of myofascial structure and tight muscle, extending of hip 
adductors, gluteals, hamstrings and hip flexors. f) Cardio vascular-based exercise programs which support the protracting factors or more unique reinforcing.

\section{Post-Operative Recovery}

Post-operative recovery can be begun with isometric activities for muscular strength and muscles around the hip joint. Early spinal activation program can likewise be started. Increment strolling utilizing time as restricting variable, expanding by five minutes out of every day can be started from second week onwards and dynamic helped practices and isokinetic program in utilitarian standing position can be fused toward the finish of second week. Useful recovery program by utilizing gymnastic ball, versatility and strength practices and the activity to further develop cardiovascular framework can be begun with from third week onwards. Early sports related explicit recovery program can be fused with the with the current recovery program in the fourth and fifth long stretches of a medical procedure. The individual can get back to typical sports by fifth or sixth week as indicated by the utilitarian reassessment.

\section{Summary}

Athletic pubalgia is a complex condition that is constrained by a few factors that should be treated with legitimate consideration and sharpness. A clinician should analyze the gathering of symptoms very right off the bat to forestall the odds of additional injury to their athletes. Ideal acknowledgment is essential if there should be an occurrence of injury determination which should be possible through exhaustive assessment of the injury. This might incorporate palpation of the spaces of concern and manual muscle testing to bar the other potential conditions. Generally, the patient will grumble about constant agony in the areas, for example, lower mid-region, pelvic locale and thighs which all transmits to the groin district. Assuming a clinician experiences such grumblings, he/she ought to look at the patient for athletic pubalgia. Nonetheless, one should know about the way that these discoveries won't affirm the analysis of athletic pubalgia, so clinicians ought to have an intensive information on different conditions which might mirror the symptoms of athletic pubalgia. Athletic pubalgia might be brought about by an repetitive eccentric overburden to the abdominal wall stabilizers of the pelvis giving delicacy over the pubic tubercle and symphysis. All the more regularly, a patient will report practice related groin torment which might be lethargic to conservative treatment. Most imaging considers have restricted capacity to analyze athletic pubalgia and is simply helpful to bar other conclusion. Patient will regularly not react to conservative treatment, so we can switch over to surgical treatment if patient doesn't react to non-usable treatment even following a month and a half. Generally quiet re-visitations of his pre-injury level inside about a month and a half get-togethers fix and inside 1 to a half year after an open fix.

\section{REFERENCES}

1. Gilmore, J. (1998). Groin pain in the soccer athlete: fact, fiction, and treatment. Clinics in sports medicine, 17(4), 787-793.

2. Swan Jr, K. G., \& Wolcott, M. (2007). The athletic hernia: a systematic review. Clinical Orthopaedics and Related Research $\AA, 455,78-87$.

3. Meyers, W. C., Foley, D. P., Garrett, W. E., Lohnes, J. H., \& Mandlebaum, B. R. (2000). Management of severe lower abdominal or inguinal pain in high-performance athletes. The American journal of sports medicine, 28(1), 2-8.

4. Hölmich, P. (2007). Long-standing groin pain in sportspeople falls into three primary patterns, a "clinical entity" approach: a prospective study of 207 patients. British journal of sports medicine, 41(4), 247-252.

5. Kachingwe, A. F., \& Grech, S. (2008). Proposed algorithm for the management of athletes with athletic pubalgia (sports hernia): a case series. Journal of orthopaedic \& sports physical therapy, 38(12), 768-781.

6. Taylor, D. C., Meyers, W. C., Moylan, J. A., Lohnes, J., Bassett, F. H., \& Garrett JR, W. E. (1991). Abdominal musculature abnormalities as a cause of groin pain in athletes: inguinal hernias and pubalgia. The American journal of sports medicine, 19(3), 239-242.

7. Preskitt, J.T. (2011). Sports Hernia: the experience of Baylor University Medical Center at Dallas. Proc (Baylo Univ Med Cent); 24(2); 89-91.

8. Nam, A., Brody, F. (2008). Management and therapy for sports hernia. J Amer Coll Surg, 206(1); 154-164.

9. Larson, C.M., Lohnes, J.H. (2002). Surgical management of athletic pubalgia. Operative Techniques in Sports Medicine, 10; 228-232.

10. Diesen, D.L., Pappas, T.N. (2007). Sports hernias. Adv Surg, 41; 177-187.

11. Meyers, W.C., Yoo, E., Devon, O.N. (2007). Understanding "sports hernia" (athletic pubalgia): The anatomic and pathophysiologic basis for abdominal and groin pain in athletes. Operative Techniques in Sports Medicine, 15; 165-177.

12. Rizk, N.N. (1980). A new description of the anterior abdominal wall in man and mammals. $J$ Anat, 131(Pt 3):373-385

13. Robinson, P., Salehi, F., Grainger, A. (2007). Cadaveric and MRI study of the musculotendinous contributions to the capsule of the symphysis pubis. AJR, 188;1306; [web] W440-W445

14. Bradshaw, C., Hölmich, P. (2006). Longstanding groin pain. In: Brukner P, Khan K (eds) Clinical sports medicine, 3rd edn. McGraw-Hill, Sydney, Australia

15. Anderson, K., Strickland, S. M., \& Warren, R. (2001). Hip and groin injuries in athletes. The American journal of sports medicine, 29(4), 521533. 
16. Joesting, D.R. (2002). Diagnosis and treatment of sportsman's hernia. Curr Sports Med Rep, 1; 121124

17. Paluska, S.A. (2005). An overview of hip injuries in running. Sports Med, 35; 991-1014.

18. Farber, A. J., \& Wilckens, J. H. (2007). Sports hernia: diagnosis and therapeutic approach. JAAOS-Journal of the American Academy of Orthopaedic Surgeons, 15(8), 507-514.

19. Ahumada, L.A., Ashruf, A., Espinosa-de-losMonteros, A. (2005). Athletic pubalgia definition and surgical treatment. Ann Plast Surg, 55; 393396.

20. Farber, A. J., \& Wilckens, J. H. (2007). Sports hernia: diagnosis and therapeutic approach. JAAOS-Journal of the American Academy of Orthopaedic Surgeons, 15(8), 507-514.

21. Geisler, P. R., \& Kelly, E. (2008). Groin pains. Training and conditioning, 18(7), 31-5.

22. Skandalakis, J. E., Gray, S. W., Skandalakis, L. J., Colborn, G. L., \& Pemberton, L. B. (1989). Surgical anatomy of the inguinal area. World journal of surgery, 13(5), 490-498.

23. Akermark, C., \& Johansson, C. (1992). Tenotomy of the adductor longus tendon in the treatment of chronic groin pain in athletes. The American journal of sports medicine, 20(6), 640-643.

24. Vix, V.A., Ryu, C.Y. (1971). The adult symphysis pubis: normal and abnormal. Am J Roentgenol Radium Ther Nucl Med, 112; 517-525

25. Verrall, G. M., Slavotinek, J. P., \& Fon, G. T. (2001). Incidence of pubic bone marrow oedema in Australian rules football players: relation to groin pain. British journal of sports medicine, 35(1), 2833.

26. Gibbon, W. W., \& Hession, P. R. (1997). Diseases of the pubis and pubic symphysis: MR imaging appearances. AJR. American journal of roentgenology, 169(3), 849-853.

27. Kunduracioglu, B., Yilmaz, C., Yorubulut, M., \& Kudas, S. (2007). Magnetic resonance findings of osteitis pubis. Journal of Magnetic Resonance Imaging: An Official Journal of the International Society for Magnetic Resonance in Medicine, 25(3), 535-539.

28. Brennan, D., O'Connell, M. J., Ryan, M., Cunningham, P., Taylor, D., Cronin, C., ... \& Eustace, S. (2005). Secondary cleft sign as a marker of injury in athletes with groin pain: MR image appearance

and interpretation. Radiology, 235(1), 162-167.

29. Meyers, W. C., Lanfranco, A., \& Castellanos, A. (2002). Surgical management of chronic lower abdominal and groin pain in high-performance athletes. Current sports medicine reports, 1(5), 301-305.

30. Gokhale, S. (2007). Three-dimensional sonography of muscle hernias. Journal of ultrasound in medicine, 26(2), 239-242.

31. Mellado, J. M., \& Del Palomar, L. P. (1999). Muscle hernias of the lower leg: MRI findings. Skeletal radiology, 28(8), 465-469.

32. LeBlanc, K. E., \& LeBlanc, K. A. (2003). Groin pain in athletes. Hernia, 7(2), 68-71.

33. Lynch, S. A., \& Renström, P. A. (1999). Groin injuries in sport. Sports medicine, 28(2), 137-144.

34. Morelli, V., \& Weaver, V. (2005). Groin injuries and groin pain in athletes: part 1. Primary care: clinics in office practice, 32(1), 163-183.

35. Overdeck, K. H., \& Palmer, W. E. (2004, February). Imaging of hip and groin injuries in athletes. In Seminars in musculoskeletal radiology (Vol. 8, No. 01, pp. 41-55). Copyright $@$ 2004 by Thieme Medical Publishers, Inc., 333 Seventh Avenue, New York, NY 10001, USA.

36. Shortt, C. P., Zoga, A. C., Kavanagh, E. C., \& Meyers, W. C. (2008, March). Anatomy, pathology, and MRI findings in the sports hernia. In Seminars in musculoskeletal radiology (Vol. 12, No. 01, pp. 054-061). (C) Thieme Medical Publishers

37. Kluin, J., den Hoed, P. T., van Linschoten, R., IJzerman, J. C., \& van Steensel, C. J. (2004). Endoscopic evaluation and treatment of groin pain in the athlete. The American journal of sports medicine, 32(4), 944-949.

38. Van Veen, R. N., de Baat, P., Heijboer, M. P., Kazemier, G., Punt, B. J., Dwarkasing, R. S., ... \& van Eijck, C. H. J. (2007). Successful endoscopic treatment of chronic groin pain in athletes. Surgical endoscopy, 21(2), 189-193.

39. Nelson, E. N., Kassarjian, A., \& Palmer, W. E. (2005). MR imaging of sports-related groin pain. Magnetic Resonance Imaging Clinics, 13(4), 727-742.

40. Lacroix, V. J., Kinnear, D. G., Mulder, D. S., \& Brown, R. A. (1998). Lower abdominal pain syndrome in national hockey league players: a report of 11 cases. Clinical journal of sport medicine: official journal of the Canadian Academy of Sport Medicine, 8(1), 5-9.

41. Macintyre, J., Johson, C., \& Schroeder, E. L. (2006). Groin pain in athletes. Current sports medicine reports, 5(6), 293-299.

42. Van Der Donckt, K., Steenbrugge, F., Van Den Abbeele, K., Verdonk, R., \& Verhelst, M. (2003). Bassini's hernial repair and adductor longus tenotomy in the treatment of chronic groin pain in athletes. pain, 36,38 . 